\title{
Hypnosis intervention for the management of pain perception during cataract surgery
}

This article was published in the following Dove Press journal: Journal of Pain Research

\section{Xiaofan Chen ${ }^{1, *}$ \\ Rongdi Yuan ${ }^{1, *}$ \\ Xiao Chen ${ }^{2}$ \\ Min Sun ${ }^{2}$ \\ Sen $\operatorname{Lin}^{2}$ \\ Jian $\mathrm{Ye}^{2}$ \\ Chunlin Chen $^{2}$}

'Department of Ophthalmology, Xinqiao Hospital, Third Military Medical University, Chongqing, 400042, China; 'Department of Ophthalmology, Institute of Surgery Research, Daping Hospital, Third Military Medical University, Chongqing, 400042, China

*These authors contributed equally to this work

Correspondence: Chunlin Chen Department of Ophthalmology, Xinqiao Hospital, Third Military Medical University, 83 Xinqiao Main Street, Chongqing 400042, China

Tel +86236875 7765

Fax +862368757767

Email chunlinc@126.com
Objective: To investigate the effectiveness of hypnosis in pain management during cataract surgery.

Methods: Male or female patients with bilateral age-related cataract who wished to have both eyes subjected to phacoemulsification surgery were preliminarily admitted. Immediately after the first-eye surgery, each patient was evaluated for pain using the visual analog scale (VAS), and patients with a VAS score $>1$ were enrolled. By using block randomization, the enrolled patients were allocated to either the treatment group, which received a hypnosis intervention before the scheduled second-eye surgery, or the control group, which did not undergo hypnosis. The levels of anxiety, pain, and cooperation were evaluated independently by the patients and the surgeon. Results: During the intraoperative pain assessment, 5\%, 34\%, 38\%, and $23 \%$ of patients in the control group reported experiencing no pain, mild pain, moderate pain, and severe pain, respectively. In contrast, in the hypnosis group, $18 \%, 56 \%, 15 \%$, and $11 \%$ of patients reported experiencing no pain, mild pain, moderate pain, and severe pain, respectively, which showed significant differences between the groups $(P<0.005)$. The evaluation of anxiety level showed that the mean score in the control group and hypnosis group was $11.77 \pm 0.32$ and $6.64 \pm 0.21$, respectively, revealing a highly significant difference between the two groups $(P<0.005)$. The assessment of patient cooperation showed that only $5 \%$ and $18 \%$ of patients in the control group and $18 \%$ and $36 \%$ of patients in the hypnosis group showed excellent and good cooperation, respectively, while $47 \%$ of patients in the control group and only $24 \%$ of patients in the hypnosis group exhibited poor cooperation, revealing significant differences between the groups $(P<0.005)$. Conclusion: Hypnosis may be considered as an auxiliary measure in cataract surgery, especially for patients who experienced obvious pain during the first-eye surgery.

Keywords: pain, patient satisfaction, cataract surgery, hypnosis

\section{Introduction}

Phacoemulsification is the most effective cataract surgery currently performed and is characterized by a smaller wound and reduced surgery time. Given the advancements in equipment and the proficiency of surgical skills, topical anesthesia is the preferred method in phacoemulsification surgery to avoid the risks associated with local (retrobulbar or peribulbar) anesthesia, such as chemosis, ecchymosis, retrobulbar hemorrhage, globe penetration, or perforation $;^{1,2}$ however, phacoemulsification with topical anesthesia is not a completely painless procedure, with patients frequently experiencing pain during the surgery. ${ }^{1,3-6}$ In a study comparing topical anesthesia and local anesthesia for phacoemulsification surgery, about 
$30.1 \%$ of patients with topical anesthesia complained of intraoperative pain, while only $6.7 \%$ of patients with local anesthesia reported intraoperative pain. ${ }^{7}$ In another study, $78.3 \%$ of patients reported experiencing pain during phacoemulsification surgery with topical anesthesia; when the intensity of pain was evaluated, the percentage of patients reporting mild, moderate, and intense pain was $35.9 \%, 25.0 \%$, and $17.4 \%$, respectively. ${ }^{5}$ Although the use of topical anesthesia may help avoid the potential complications of local anesthesia, the possibility of the eye moving or closing due to pain may represent a significant handicap; thus, preoperative administration of analgesics or the use of an additional sedative measure may increase patient comfort and cooperation.

Hypnosis is a method of psychotherapy that can modulate the phenomenological aspects of conscious experience, relieve pain, relax the body, and reduce anxiety. ${ }^{8,9} \mathrm{~A}$ limited number of studies have demonstrated the efficacy of clinical hypnosis in the treatment of pain and anxiety in severe diseases. ${ }^{10-12}$ In the context of anesthesia, hypnosis can be used alone or in combination with the drugs typically used in anesthetic regimens, and increasing evidence supports the value of hypnosis in reducing patient stress and anxiety and inducing relaxation. ${ }^{13,14}$

In this study, we discuss the effectiveness of hypnosis in alleviating pain associated with cataract surgery.

\section{Methods}

\section{Patients}

Male or female patients, aged 50-85 years, with bilateral agerelated cataract and who wished to have phacoemulsification surgery in both eyes were preliminarily admitted. Immediately after the first-eye surgery, each patient was evaluated for pain using the visual analog scale (VAS), and patients with a VAS score $>1$ were enrolled. Using block randomization, the enrolled patients were allocated to either the treatment group, which received hypnosis intervention before the scheduled second-eye surgery, or the control group, which did not undergo hypnosis.

\section{Treatment}

Patients in the treatment group were managed by a hypnotherapist half an hour before the second-eye surgery. After explaining the hypnosis procedure, the hypnotherapist initiated a 10 - to 15 -minute short hypnosis session using a technique described by Erickson; ${ }^{15}$ after the hypnosis session, the patient's anxiety for the forthcoming surgery was evaluated, and a standard phacoemulsification surgery under topical anesthesia was performed by the same surgeon. The topical anesthesia used was $0.5 \%$ proparacaine hydrochloride drops (Alcaine, Alcon Pharma; Puurs, Belgium) which was applied to the ocular surface twice at 2- to 3-minute intervals. In cases where patients experienced unbearable pain and were uncooperative, an additional dose of $2 \%$ lidocaine was administered by sub-Tenon's injection. The patients in the control group underwent the same procedures except for the hypnosis session. Patients who had any of the following were excluded from the study: complicated surgeries, such as zonular dialysis or posterior capsule rupture; a history of previous eye surgery; evidence of injury or inflammation; nystagmus; communication or cooperation difficulties; any prior experience of using hypnosis; conditions of psychosis, such as hallucinations, delusions, or depression; or excessive smoking and drinking habit. The surgeon was blinded to the group allocations until the entire study was completed. Upon completion of the surgery, the patients received oral instructions to guide their return to consciousness, and pain and cooperation levels were subsequently evaluated by the patients and the surgeon separately.

Patient pain was evaluated after the surgery using a VAS ruler as follows: 0 point, no pain; $1-3$ points, mild pain; and $>3$ points, moderate-to-severe pain (Figure 1).

Patient cooperation was graded from 0 (no event) to 3 (obvious eye and head movement and/or lid squeezing) by the surgeon after the surgery. The grade was classified as follows: 0, excellent cooperation (no events); 1 , good cooperation (eyelid squeezing); 2, sufficient cooperation (globe movement and eyelid squeezing); 3 , poor cooperation (head movement, globe movement, and eyelid squeezing).

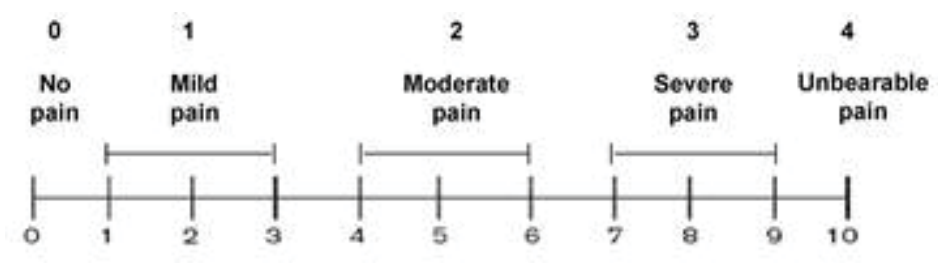

Figure I Pain score chart. 0, no pain; I-3, mild pain; 4-6, moderate pain; 7-9, severe pain; I0, unbearable pain. 
Patient anxiety was evaluated before surgery using the Amsterdam Preoperative Anxiety and Information Scale (APAIS). The test involved a six-item questionnaire. Four items in the questionnaire were used to measure preoperative anxiety, and two items were used to measure the need for information. The items were rated on a 5-point Likert scale from "not at all" to "extremely". Each question was scored from 1 to 5 . The total anxiety scale score ranged from 4 to 20. The information demand scale score ranged from 2 to 10 .

This study was approved by the Third Affiliated Hospital Institutional Review Board, Third Military Medical University, Chongqing, China, and all patients provided written informed consent. The clinical trial registration number is ChiCTR1800014711.

\section{Statistical analysis}

Quantitative data were compared between the two groups via $t$-test (normal distribution) and the Wilcoxon rank sum test (non-normal distribution). The $\chi^{2}$ test was used to compare categorical variables. The Spearman rank correlation was used for nonparametric data. Statistical analysis was performed using SPSS 13.0 (SPSS Inc., Chicago, IL, USA). The sample size for the study was based on the results from an earlier study; two-tailed $t$-tests were conducted with $\alpha=0.05$ and $\beta=0.1$, and it was determined that at least 50 patients were required for each group. Taking patient discontinuation into consideration, as well as possible complications in the surgery, a total of 120 patients were chosen to ensure sufficient enrollment.

\section{Results}

A total of 120 patients were enrolled and formed the intentto-treat population, with 60 patients randomized to each study group. Among the enrolled patients, three in the treatment group and two in the control group did not undergo their second-eye surgery for health or other reasons. In addition, two patients from each group were excluded from the study because complications occurred and lidocaine was used in the surgery. Hence, 111 patients (48 men and 53 women, 55 in the treatment group and 56 in the control group) between 50 and 82 years (median 64 years) were included in the final evaluation. Baseline characteristics were well balanced between the two groups (Table 1 and Figure 2).

During the intraoperative pain assessment using the VAS, $5 \%, 34 \%, 38 \%$, and $23 \%$ of patients in the control group reported having no pain, mild pain, moderate pain, and severe pain, respectively. In contrast, in the hypnosis group, $18 \%$, $56 \%, 15 \%$, and $11 \%$ of patients reported having no pain, mild
Table I Patient characteristics

\begin{tabular}{llll}
\hline Parameter & Hypnosis & Control & $P$-value \\
\hline Patients $(\mathrm{n})$ & 55 & 56 & - \\
Mean age (years) \pm SD & $64.1 \mathrm{I} \pm 5.74$ & $64.66 \pm 5.07$ & 0.59 \\
Sex (male/female) & $25 / 30$ & $27 / 29$ & 0.85 \\
\hline
\end{tabular}

pain, moderate pain, and severe pain, respectively, which showed significant differences between the groups $(P<0.005)$ (Table 2 and Figure 3).

Patient anxiety levels were evaluated using the APAIS. A highly significant difference was noted between the control group and the hypnosis group $(P<0.005)$. The mean score of the control group was 11.77 (SD 0.32), and the mean score of the hypnosis group was 6.64 (SD 0.21). Results from the patient anxiety assessments are presented in Table 3 and Figure 4.

The cooperation assessment revealed that only $5 \%$ and $18 \%$ of patients in the control group exhibited excellent and good cooperation, respectively, whereas $47 \%$ of patients exhibited poor cooperation. In contrast, in the hypnosis group, $18 \%$ and $36 \%$ of patients exhibited excellent and good cooperation, respectively, whereas $24 \%$ exhibited poor cooperation, which showed significant differences among the four groups $(P<0.005)$. The results of the patient cooperation assessments are presented in Table 4 and Figure 5.

There was a significant positive correlation between the VAS and cooperation, both in the hypnosis group and in the control group $(r=0.414, P<0.005 ; r=0.596, P<0.005)$. However, there was no significant correlation between the VAS and the anxiety analysis in either group.

\section{Discussion}

In conventional cataract surgery, a skilled doctor can perform a successful phacoemulsification surgery in approximately 10 minutes, and topical anesthesia is thus often used to save time; however, topical anesthesia is not always completely effective for relieving pain and anxiety in phacoemulsification surgery. Most recent studies have focused on determining whether the pain experienced during the second-eye surgery is greater than or equal to that experienced during the first-eye surgery, ${ }^{16-19}$ but few researchers have focused on reducing patient pain during the cataract surgery under topical anesthesia. In fact, if the patient experienced pain during the first-eye surgery, particularly obvious pain, he/ she will inevitably experience pain and anxiety during the second-eye surgery if only local anesthesia is used without any auxiliary sedative measures. 


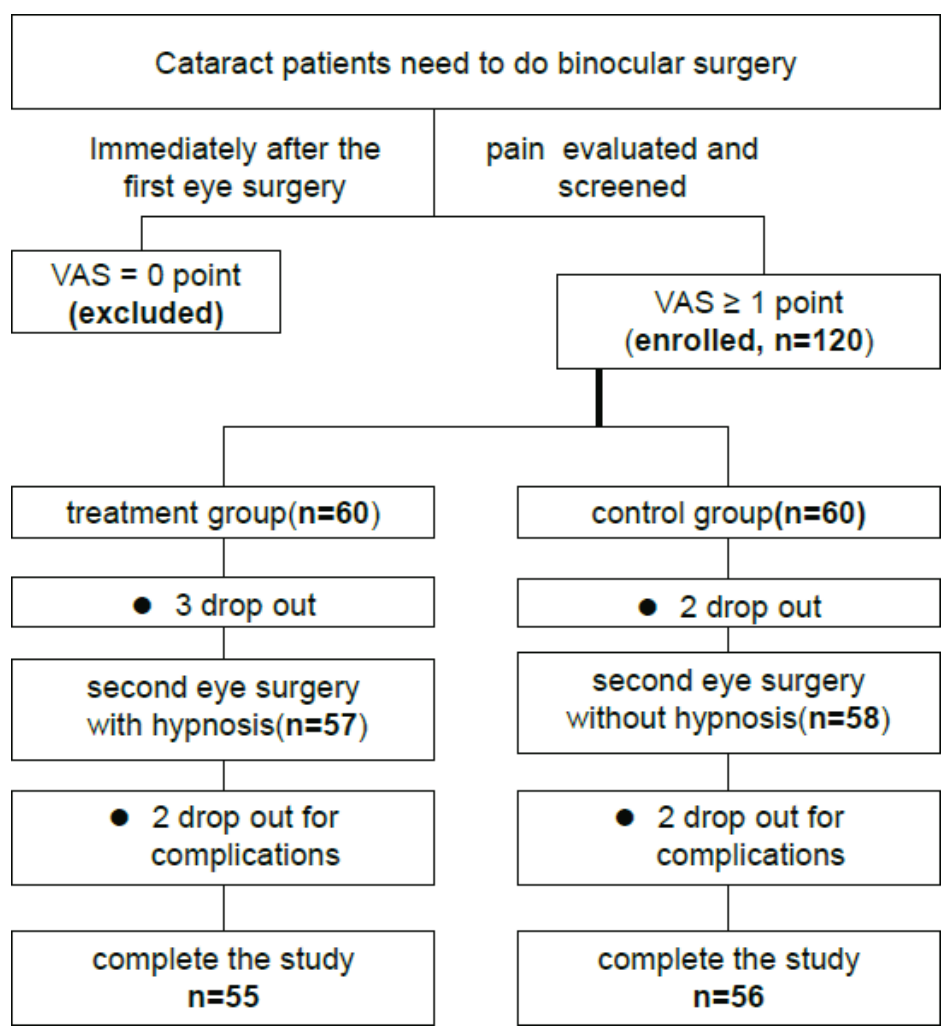

Figure 2 Study flow chart.

Abbreviation: VAS, visual analog scale.

Table 2 Patient VAS scores

\begin{tabular}{|c|c|c|c|c|c|c|c|}
\hline \multirow[t]{2}{*}{ Groups } & \multirow[t]{2}{*}{$\mathbf{n}$} & \multicolumn{4}{|c|}{ VAS during phacoemulsification surgery, $\mathbf{n}(\%)$} & \multirow[t]{2}{*}{$\chi^{2}$} & \multirow[t]{2}{*}{$P$-value } \\
\hline & & 0 & $\mathrm{I}-2$ & 2-3 & $3-4$ & & \\
\hline Hypnosis & 55 & $10(18 \%)$ & $31(56 \%)$ & $8(15 \%)$ & $6(11 \%)$ & 15.557 & 0.001 \\
\hline Control & 56 & $3(5 \%)$ & $19(34 \%)$ & 21 (38\%) & $13(23 \%)$ & & \\
\hline
\end{tabular}

Abbreviation: VAS, visual analog scale.

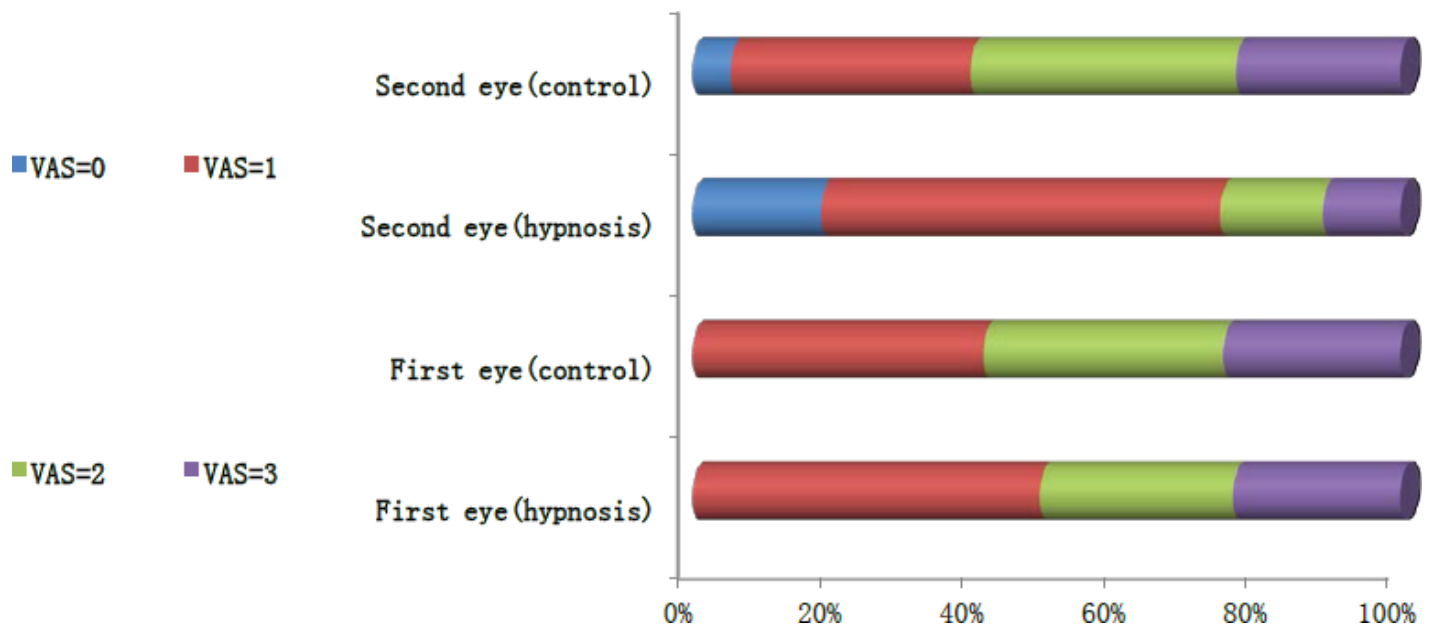

Figure 3 Extent of patient pain with or without hypnosis.

Abbreviation: VAS, visual analog scale. 
Hypnosis has been used in ophthalmic surgery for many years, and several cases of successful hypnosis application have been reported..$^{20,21}$ However, these reports mainly focused on the ability of hypnosis to reduce medication use and increase patient satisfaction or described its use in high-risk patients in unusual situations. ${ }^{22-24}$ In the present study, we assessed whether hypnosis can assist in alleviating cataract patient pain and anxiety and improve cooperation in the second-eye surgery. In total, our results showed that patients in the hypnosis group exhibited remarkably improved results, though hypnosis combined with topical anesthesia was not able to eliminate pain for all patients, which may be reasonable given individual differences in the hypnotic effect and pain sensitivity. Overall, many patients in the hypnosis group presented with reduced pain, lower anxiety, and increased cooperation.

Our study revealed two main conclusions. First, with hypnosis therapy, patients with a higher VAS score in their first-eye surgery showed a remarkable decrease in VAS score in their second-eye surgery (data not shown). These

Table 3 Patient anxiety evaluation

\begin{tabular}{lllll}
\hline Groups & $\mathbf{n}$ & $\begin{array}{l}\text { Anxiety before the } \\
\text { phacoemulsification } \\
\text { surgery (mean } \pm \text { SD) }\end{array}$ & $\boldsymbol{t}$ & P-value \\
\hline Hypnosis & 55 & $6.64 \pm 0.21$ & 13.21 & $<0.0001$ \\
Control & 56 & $11.77 \pm 0.32$ & & \\
\hline
\end{tabular}

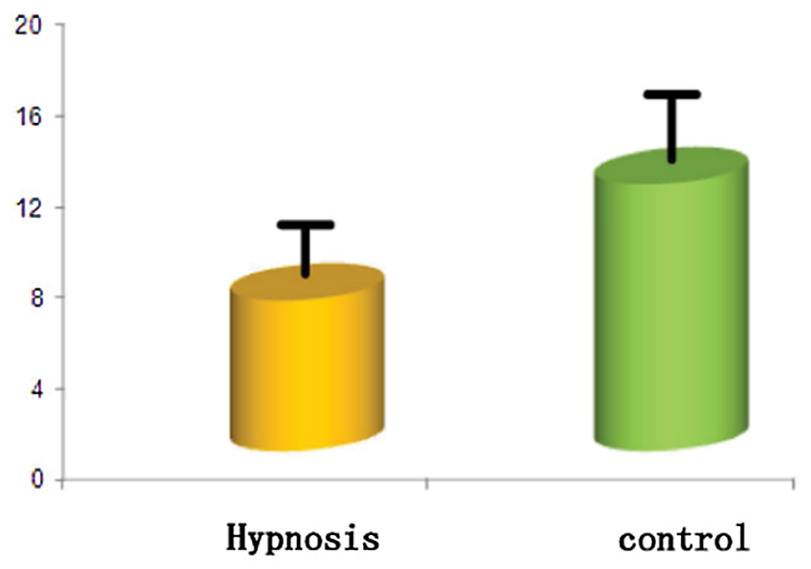

Figure 4 Anxiety levels in patients with or without hypnosis.

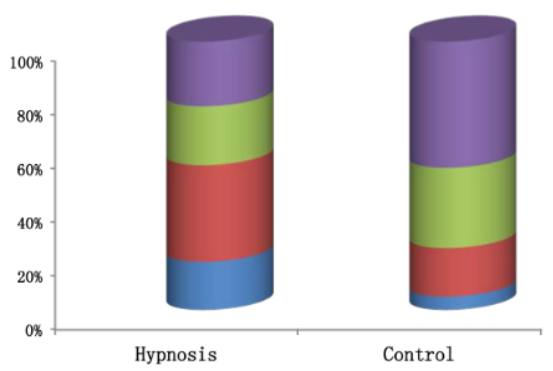

Figure $\mathbf{5}$ Cooperation levels in patients with or without hypnosis.

data suggest that hypnosis was able to help alleviate pain, especially for patients who experienced obvious pain during the first-eye surgery. Second, patients VAS score was positively associated with cooperation score, indicating that patients who experience less pain demonstrate better cooperation.

\section{Conclusion}

To the best of our knowledge, this is the first study investigating the effects of hypnosis on the management of pain during cataract surgery in the second eye. Although our data must be interpreted with caution given the high variability in hypnosis techniques, the lack of standard scales to indicate hypnotic susceptibility, individual differences to hypnotic susceptibility, and the small number of patients included in the study, our results clearly demonstrated that hypnosis may be considered as an auxiliary measure for cataract surgery in the second eye, especially for patients who experienced pain during the first-eye surgery.

\section{Acknowledgments}

The authors would like to thank all the patients for graciously participating in the study and the reviewers for their valuable comments.

\section{Author contributions}

Chunlin Chen and Jian Ye designed the study and conducted the analyses. Xiaofan Chen, Rongdi Yuan, Xiao Chen, and Min Sun performed the experiments. Xiaofan Chen and Sen Lin wrote the manuscript. All authors read and approved the final manuscript.

Table 4 Patient cooperation scores

\begin{tabular}{lllllll}
\hline Groups & $\mathbf{n}$ & \multicolumn{2}{l}{ Cooperation during phacoemulsification surgery, $\mathbf{n}(\%)$} & \multirow{2}{*}{$\chi^{\mathbf{2}}$} & P-value \\
\cline { 3 - 6 } & & $\mathbf{0}$ & $\mathbf{I}$ & $\mathbf{2}$ & $\mathbf{3}$ & 12.944 \\
\hline Hypnosis & 55 & $10(18 \%)$ & $20(36 \%)$ & $12(22 \%)$ & $13(24 \%)$ & 0.005 \\
Control & 56 & $3(5 \%)$ & $10(18 \%)$ & $16(30 \%)$ & $27(47 \%)$ & \\
\hline
\end{tabular}




\section{Disclosure}

The authors report no conflicts of interest in this work.

\section{References}

1. Ezra DG, Allan BD. Topical anaesthesia alone versus topical anaesthesia with intracameral lidocaine for phacoemulsification. Cochrane Database Syst Rev. 2007;18(3):CD005276.

2. Nanji KC, Roberto SA, Morley MG, Bayes J. Preventing Adverse Events in Cataract Surgery: Recommendations From a Massachusetts Expert Panel. Anesth Analg. 2018;126(5):1537-1547.

3. Hosoda Y, Kuriyama S, Jingami Y, Hattori H, Hayashi H, Matsumoto M. A comparison of patient pain and visual outcome using topical anesthesia versus regional anesthesia during cataract surgery. Clin Ophthalmol. 2016;10:1139-1144.

4. Ezra DG, Nambiar A, Allan BD. Supplementary intracameral lidocaine for phacoemulsification under topical anesthesia. A metaanalysis of randomized controlled trials. Ophthalmology. 2008;115(3): 455-487.

5. Dada1 Z, Borazan M, Öncel Acır N. Pain Perception in Phacoemulsification with Topical Anesthesia and Evaluation of Factors Related with Pain. Turk J Ophthalmol. 2016;46(4):151-155.

6. Kongsap P, Wiriyaluppa C. A comparison of patient pain during cataract surgery with topical anesthesia in Prechop Manual Phacofragmentation versus phacoemulsification. J Med Assoc Thai. 2006;89(7):959-966.

7. Uusitalo RJ, Maunuksela EL, Paloheimo M, Kallio H, Laatikainen L. Converting to topical anesthesia in cataract surgery. J Cataract Refract Surg. 1999;25(3):432-440.

8. Kendrick C, Sliwinski J, Yu Y, et al. Hypnosis for Acute Procedural Pain: A Critical Review. Int J Clin Exp Hypn. 2016;64(1):75-115.

9. Lynn SJ, Laurence JR, Kirsch I, Hypnosis, suggestion, and suggestibility: an integrative model. Am J Clin Hypn. 2015;57(3):314-329.

10. Barbero U, Ferraris F, Muro M, Budano C, Anselmino M, Gaita F. Hypnosis as an effective and inexpensive option to control pain in transcatheter ablation of cardiac arrhythmias. J Cardiovasc Med (Hagerstown). 2018;19(1):18-21.
11. Jafarizadeh H, Lotfi M,Ajoudani F, Kiani A, Alinejad V. Hypnosis for reduction of background pain and pain anxiety in men with burns: A blinded, randomised, placebo-controlled study. Burns. 2018;44(1):108-117.

12. Bhatt RR, Martin SR, Evans S, et al. The effect of hypnosis on pain and peripheral blood flow in sickle-cell disease: a pilot study. J Pain Res. 2017;10:1635-1644.

13. Facco E. Hypnosis and anesthesia: back to the future. Minerva Anestesiol. 2016;82(12):1343-1356.

14. Wobst AH. Hypnosis and surgery: past, present, and future. Anesth Analg. 2007;104(5):1199-1208.

15. Erickson MH. Further clinical techniques of hypnosis: utilization techniques. 1959. Am J Clin Hypn. 2009;51(4):341-362.

16. Sharma NS, Ooi JL, Figueira EC, et al. Patient perceptions of second eye clear corneal cataract surgery using assisted topical anaesthesia. Eye (Lond). 2008;22(4):547-550.

17. Ursea R, Feng MT, Zhou M, Lien V, Loeb R. Pain perception in sequential cataract surgery: comparison of first and second procedures. $J$ Cataract Refract Surg. 2011;37(6):1009-1014

18. Hari-Kovacs A, Lovas P, Facsko A, Crate ID. Is second eye phacoemulsification really more painful? Wien Klin Wochenschr. 2012;124(15-16):516-519.

19. Akkaya S, Özkurt YB, Aksoy S, Kökçen HK. Differences in pain experience and cooperation between consecutive surgeries in patients undergoing phacoemulsification. Int Ophthalmol. 2017;37(3):545-552.

20. Lewenstein LN. Hypnosis as an anesthetic in pediatric ophthalmology. Anesthesiology. 1978;49(2):144-145.

21. John ME Jr, Parrino JP. Practical hypnotic suggestion in ophthalmic surgery. Am J Ophthalmol. 1983;96(4):540-542.

22. Lewenstein LN, Iwamoto K, Schwartz H. Hypnosis in high risk ophthalmic surgery. Ophthalmic Surg. 1981;12(1):39-41.

23. Kiss G, Butler J. Hypnosis for cataract surgery in an American Society of Anesthesiologists physical status IV patient. Anaesth Intensive Care. 2011;39(6):1139-1141.

24. Agard E, Pernod C, El Chehab H, Russo A, Haxaire M, Dot C. A role for hypnosis in cataract surgery: Report of 171 procedures. J Fr Ophtalmol. 2016;39(3):287-291.
Journal of Pain Research

\section{Publish your work in this journal}

The Journal of Pain Research is an international, peer reviewed, open access, online journal that welcomes laboratory and clinical findings in the fields of pain research and the prevention and management of pain. Original research, reviews, symposium reports, hypothesis formation and commentaries are all considered for publication.

\section{Dovepress}

The manuscript management system is completely online and includes a very quick and fair peer-review system, which is all easy to use. Visit http://www.dovepress.com/testimonials.php to read real quotes from published authors. 